\title{
Indoor Localisation Using a Context-Aware Dynamic Position Tracking Model
}

\author{
Montserrat Ros, ${ }^{1}$ Joshua Boom, ${ }^{1}$ Gavin de Hosson, ${ }^{1}$ and Matthew D’Souza ${ }^{2}$ \\ ${ }^{1}$ School of Electrical, Computer and Telecommunications Engineering, University of Wollongong, Wollongong, NSW 2522, Australia \\ ${ }^{2}$ CSIRO ICT Centre, Brisbane, QLD 4069, Australia \\ Correspondence should be addressed to Montserrat Ros, montse@uow.edu.au
}

Received 31 July 2011; Revised 14 October 2011; Accepted 1 December 2011

Academic Editor: Jinling Wang

Copyright (C) 2012 Montserrat Ros et al. This is an open access article distributed under the Creative Commons Attribution License, which permits unrestricted use, distribution, and reproduction in any medium, provided the original work is properly cited.

\begin{abstract}
Indoor wireless localisation is a widely sought feature for use in logistics, health, and social networking applications. Low-powered localisation will become important for the next generation of pervasive media applications that operate on mobile platforms. We present an inexpensive and robust context-aware tracking system that can track the position of users in an indoor environment, using a wireless smart meter network. Our context-aware tracking system combines wireless trilateration with a dynamic position tracking model and a probability density map to estimate indoor positions. The localisation network consisted of power meter nodes placed at known positions in a building. The power meter nodes are tracked by mobile nodes which are carried by users to localise their position. We conducted an extensive trial of the context-aware tracking system and performed a comparison analysis with existing localisation techniques. The context-aware tracking system was able to localise a person's indoor position with an average error of $1.21 \mathrm{~m}$.
\end{abstract}

\section{Introduction}

The next generation of pervasive media applications, mobile social networking, and location-based services are increasingly reliant on accurate position localisation. Localisation for indoor environments has many applications for pervasive media. Low-powered or green efficient and inexpensive localisation will become important for the next generation of pervasive media applications that operate on battery-constrained mobile platforms.

Current localisation techniques depend on using sensing infrastructure already present in the environment such as visual markers, wireless LAN hotspots, cellular networks, or Global Position Systems' (GPS) satellite coverage. The popular use of GPS has led to a variety of mobile location-based services applications such as social networking, street map guide, or asset tracking. Recently, there has been great interest in localisation for indoor navigation applications. Indoor environments cause multipath interference to wireless communications because of the presence of physical obstacles such as metal beams or walls. Hence, this causes outdoor Radio-Frequency- (RF-) based localisation technologies such as GPS to function inaccurately indoors because of signal degradation. Other RF localisation methods such as Received Signal Strength or Time of Arrival also experience inaccuracies and reliability issues when operating indoors.

Wireless infrastructure that is currently used for both indoor and outdoor localisation, tends to be computationally intensive with high power consumption. Wireless sensor networks are an alternative form of wireless infrastructure that can be used for localisation but also operate at low power. Wireless sensor networks are used for a sensing and actuation applications including smart metering. As energy usage monitoring becomes an important lifestyle factor for workplaces and households, wireless smart metering networks will be more widely used. Wireless smart meters are being incorporated into new buildings for climate control and to improve power usage efficiency. Wireless smart metering infrastructure can potentially be used for low-powered indoor and outdoor localisation.

We designed and developed a wireless localisation tracking system that tracked people indoors. Our wireless localisation system used a low-powered wireless smart metering network infrastructure which consisted of power metering 
nodes placed at predetermined coordinates in a building level. The power metering nodes were used to determine the coordinates of the user within the region covered by the localisation network. Our wireless localisation tracking system consisted of users carrying a mobile node to triangulate their current position. A mobile phone interface was also developed to allow users to view their location.

One of the main drawbacks of wireless indoor localisation is reduced accuracy due to multipath fading and other Radio Frequency (RF) interferences. We found that using only received signal strength or other wireless channel propagation properties was not suitable for tracking users in real time, due to the lengthy time taken to calibrate for channel propagation parameters. To overcome this, we developed a context-aware tracking model for tracking people within a building. The context-aware tracking model incorporated "awareness" of the physical context of the surrounding environment (indoor building floor plans). Odometry information such as the estimated speed was also used to predict the next position of user. We extensively evaluated our system and investigated the following aspects:

(i) use of a low-powered, inexpensive smart metering network for indoor localisation and tracking,

(ii) analysis of the wireless indoor channel propagation on position accuracy,

(iii) development and testing of a context-aware tracking algorithm,

(iv) comparison of the accuracy of the context-aware tracking model with other wireless localisation technologies and protocols.

This paper is organised into 6 sections. Section 2 presents a review of related work. Sections 3 and 4 discuss the implementation of the context-aware tracking system. Section 5 presents the evaluation findings and analysis of the system. Conclusions and further areas of investigation are discussed in Section 6.

\section{Related Work}

Different types of wireless technologies, such as GPS, have been investigated for outdoor and indoor location systems. Unfortunately, GPS is not suitable for indoor use, and this has led to research into the use of other wireless technologies including UWB [1], ultrasonic and GSM [2] platforms. Regulations are not clear for the use of UWB, and ultrasonic location detection still requires RF transceivers. GSM uses existing cellular infrastructure; however, accurate position resolution indoors is difficult.

Received Signal Strength Indicators (RSSIs) are used for indoor and outdoor localisation, as outlined by Seco et al. [3]. The most common RSSI localisation techniques are RSSI Fingerprinting, RSSI triangulation and trilateration. RSSI Fingerprinting identifies specific positions with RSSI values, while RSSI triangulation, and trilateration associate RSSI with distance or angular trajectory between receiver and known transmitter positions in order to localise [3, 4]. Hightower et al. [5] describe the PlaceLab geophysical location system in which users can determine their position in an urban environment. PlaceLab was an RSSI Fingerprinting technique that used wireless LAN hotspots and GSM broadcast towers to determine a user's position. The PlaceLab software used a database of known wireless LAN hotspots and GSM broadcast towers. The PlaceLab software can be used with a PDA or laptop with wireless LAN or GSM connectivity. Localisation accuracy is stated as being less then GPS, with $20-25 \mathrm{~m}$ using wireless LAN and $100 \mathrm{~m}$ to $150 \mathrm{~m}$ for GSM broadcast towers. A similar technique of using RSSI is employed by the power meter node network.

A classical case of using wireless beacons for navigation is presented by Want et al. [6]. The Active Badge project achieved a 5-10 $\mathrm{m}$ accuracy using infrared. The main drawback of this platform is that it required line of sight between beacons. An extension of the Active Badge Project was the ORL location system by Ward et al. [7] which developed a prototype network of ultrasonic beacons to perform realtime tracking of tagged mobile devices in an office environment. Other ultrasonic location systems such as the Cricket Mote [8] and the system by McCarthy et al. [9] describe how a network of ultrasonic beacons using time of flight analysis can determine distance position locations.

Klingbeil and Wark [10] developed a wireless sensor network for monitoring human motion and position in an indoor environment. Mobile nodes with inertial and heading sensors were worn by a person inside a building. A MonteCarlo-based localisation algorithm that used a person's heading, indoor map information, and static node positions was developed and tested. One of the problems with this approach is the tedious sensor calibration required before use.

\section{Indoor Localisation Network}

Wireless smart meters are used primarily to monitor energy consumption and also for home automation applications. Smart metering protocols include the ZigBee protocol, Advanced Meter Infrastructure [15], Dash7 [16], and wireless M-Bus [17]. Wireless smart meter networks are designed to operate as short range networks like a home and or a largescale "neighbourhood" area network. We used the ZigBee/ 802.15.4 wireless communications protocol to implement our smart meter network. ZigBee is a low data rate wireless communications protocol that can operate on devices with limited computing or power resources and cater for large networks of active devices [18].

Supported ZigBee features include Mesh Networking, 64 bit address, data rates: $20 \mathrm{kbps}$ to $250 \mathrm{kbps}$ and simple application profiles. ZigBee operates in the unlicensed ISM $2.4 \mathrm{GHz}$ or $915 \mathrm{MHz}$ frequency band [18]. Current ZigBee protocol radio transceivers have a large indoor range, up to $100 \mathrm{~m}$. The use of RSSI allowed the ZigBee protocol to be adapted for use in the indoor localisation network. Each ZigBee transceiver has a 64 bit ID address which allows a ZigBee network to handle a large number of active nodes.

The localisation network as seen in Figure 1 consisted of three types of nodes: coordinator, power meter, and mobile. Mobile nodes were carried by users to determine their current location. The power meter nodes are used to determine 


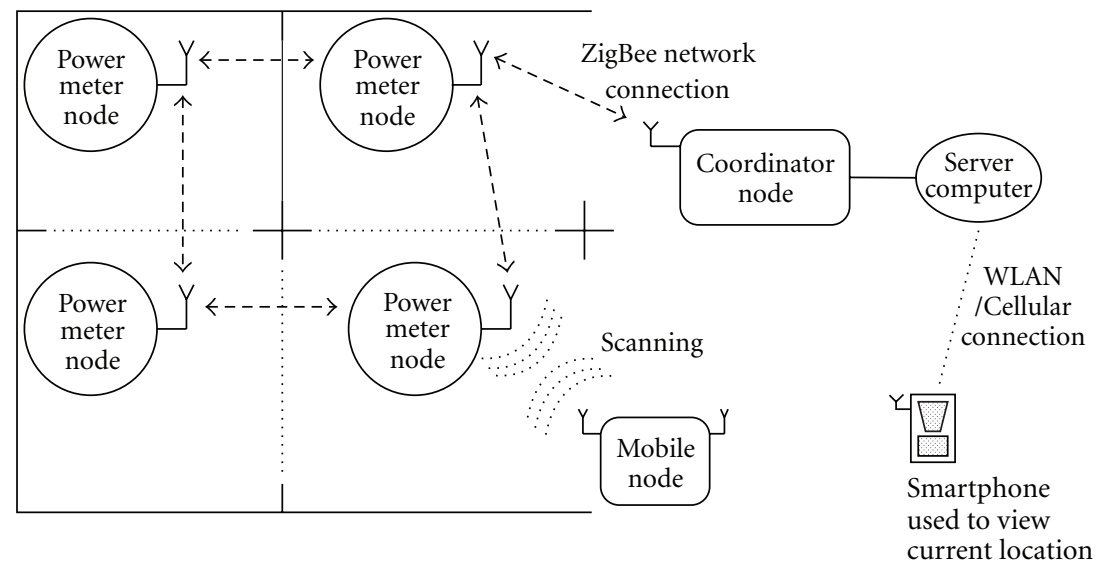

FIGURE 1: Overview of wireless smart metering network for context-aware tracking.

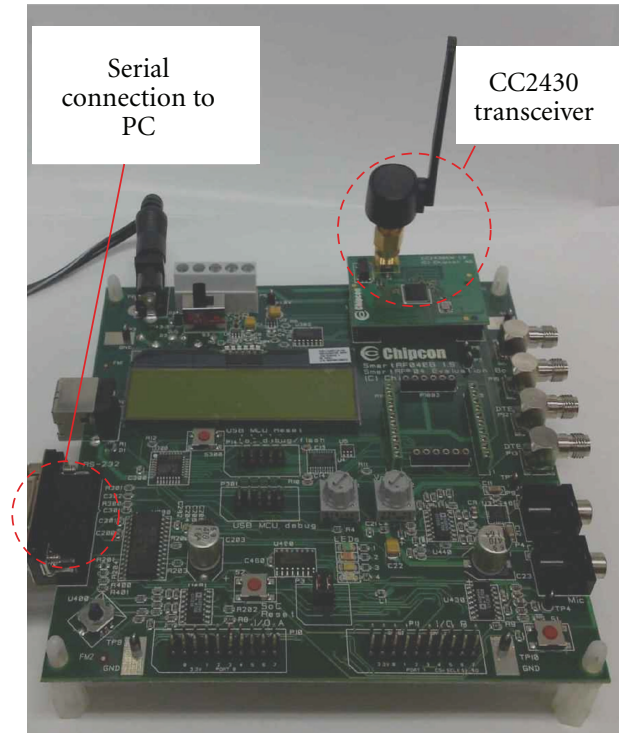

Figure 2: Coordinator node platform.

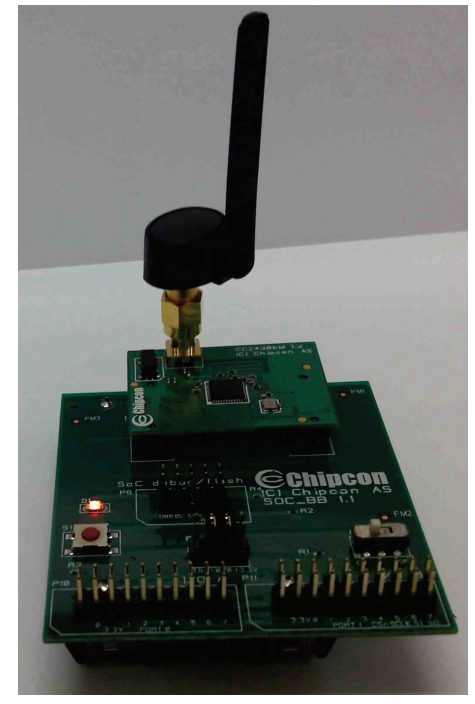

Figure 3: Power meter and mobile node platform. a mobile nodes position via trilateration. The server connected to the coordinator node displays the current positions of the mobile nodes on a building floor plan.

3.1. Coordinator Node. The coordinator node, seen in Figure 2, is used to receive the location coordinates of each mobile node. The coordinator node communicates with the mobile node via the ZigBee mesh routing connection using the power meter nodes. The mobile nodes' positions received by the coordinator can be viewed using the Z-Location graphical user interface [19]. It displays the current locations of mobile nodes on a building floor plan. The coordinator node was implemented with a CC2430 ZigBee/802.15.4 module on a SmartRF development board [19]. The coordinator node is connected by a serial connection to a server computer. The server computer tracks the position of the mobile node using the context-aware tracking process, as described in later sections. The coordinator node is also powered by standard mains electricity.

3.2. Power Meter Node. The power meter node, without power monitoring sensors, seen in Figure 3, communicates to the coordinator node via a ZigBee network connection. The position of each power meter node is known by the coordinator node. The power meter nodes are used by the mobile nodes for trilateration. The power meter node was implemented using the CC2430 ZigBee/802.15.4 wireless transceiver module from Texas Instruments [20]. Each CC2430 module has a unique 64 bit network address used as the power meter node's identifier.

3.3. Mobile Node. The function of a mobile node is to determine a user's position using received signal strength. The mobile node detects power meter nodes in near proximity. The mobile node uses the received signal strength from nearby power meter nodes to calculate its position. The predicted position is then transmitted to the coordinator nodes via 


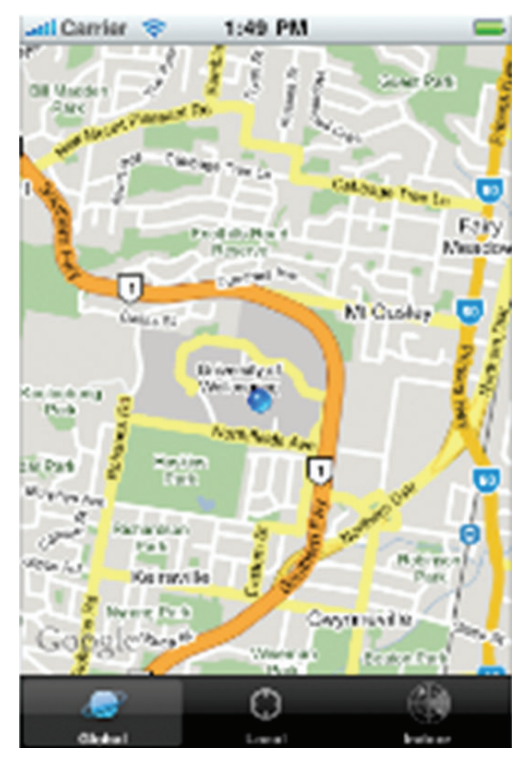

(a)

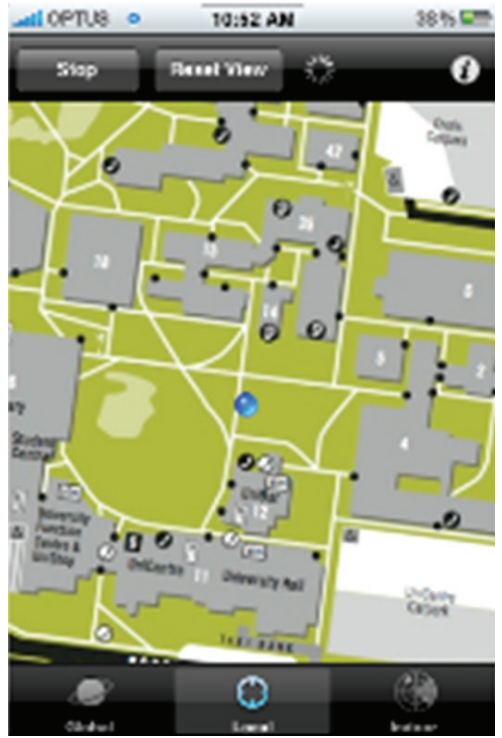

(b)

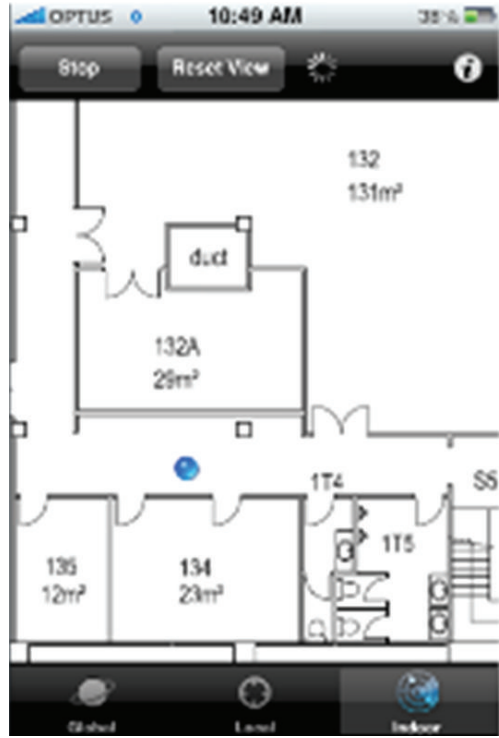

(c)

FIGURE 4: Mobile phone graphical user interface.

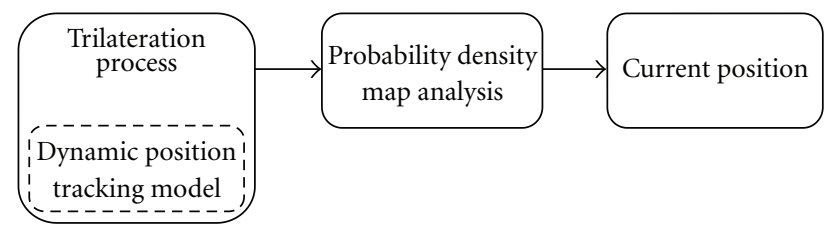

Figure 5: Overview of context-aware tracking process.

the power meter node network. The mobile node was implemented using the CC2431 ZigBee/802.15.4 Location Engine Module [20].

3.4. Mobile Phone User Interface. A mobile-phone-based graphical user interface was developed for the iPhone platform to allow users to monitor their indoor position, using the context-aware tracking system, via a cellular or wireless LAN link to the Server computer. A screenshot showing the map interface for indoor/outdoor areas can be seen in Figure 4.

\section{Context-Aware Tracking}

The context-aware tracking process calculated a person's position in real time using trilateration position estimation and a probability density map. Figure 5 shows an overview of the context-aware tracking process. First, the trilateration position estimation process is used to predict where a mobile node is located. The trilateration process used radio received signal-strength-based range-distance estimations and a Dynamic Position Tracking Model (DPTM) to compute an approximate position. A probability density map was used to determine if the position approximation was valid by using the context-aware information from the floor plan of the indoor environment.

4.1. Trilateration Position Estimation. The trilateration position estimation used the estimated range distances between the mobile node and the surrounding power meter node network to calculate the mobile node's approximate position. The range distance estimator is first used to approximate the range distance between the mobile node and the power meter nodes. The estimated range distances are then processed by DPTM to predict a set of range distances based on human motion factors such as walking speed. The trilateration algorithm used the predicted range distances between the mobile and power meter nodes to estimate the coordinates of the mobile node.

4.1.1. Range Distance Estimator. The range distances are approximated using the RSSI and the coordinates of the power meter nodes of the indoor localisation network. Figure 6 shows how the mobile nodes interact with the power meter nodes. The mobile node periodically transmitted RSSI Measure messages to the nearest power meter nodes in range (Figure 6(a)). The power meter nodes use the RSSI Measure messages to calculate the received signal strength indicator value. Five messages are used to calculate an averaged RSSI value.

As seen in Figure 6(b), once a series of RSSI Measure messages has been transmitted, the mobile node will then transmit an RSSI and Position Request message to all power meter nodes in range. Each power meter node will then respond with its calculated RSSI value and position. A minimum of three power meter nodes must be in range of 


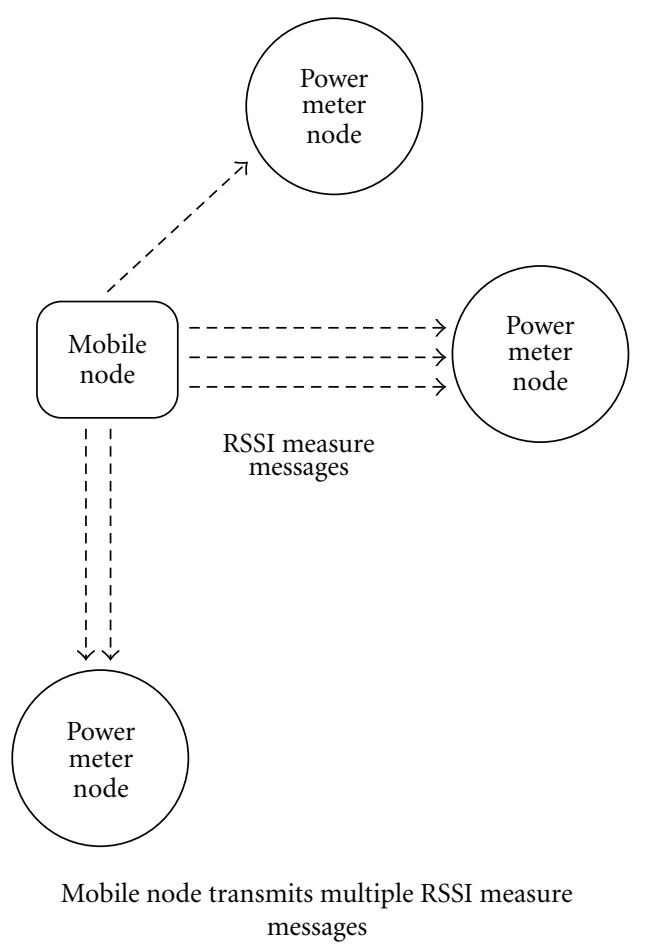

(a)

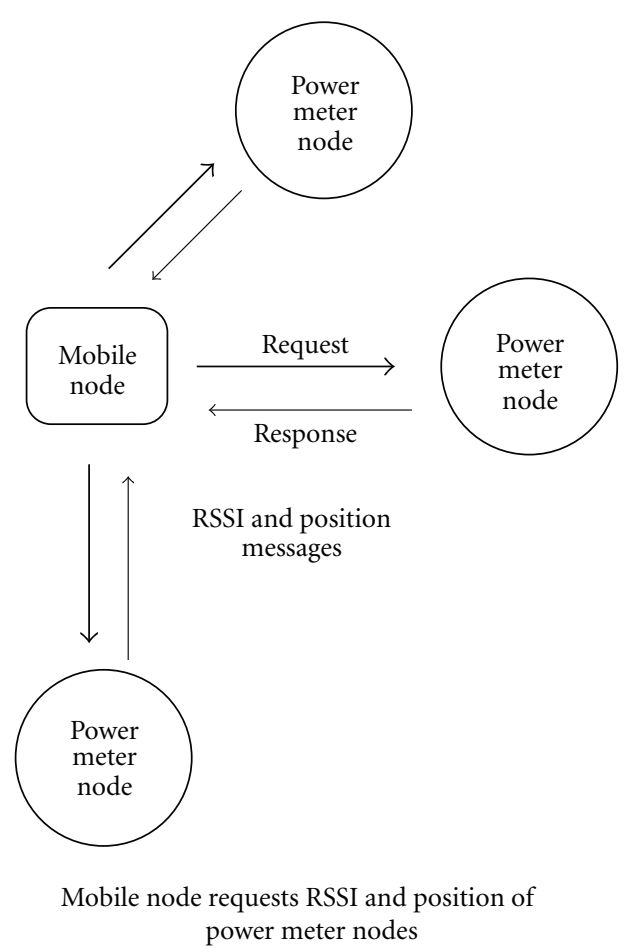

(b)

FIgURE 6: Mobile node received signal strength measuring process.

the mobile node in order to accurately approximate its position. If more than three power meter nodes are detected, the nodes with the strongest RSSI values are used. Power meter nodes with weak RSSI values were found to cause inaccuracies when calculating the range-distance estimate.

The RSSI value from the power meter node is used to calculate the range distance between the mobile and power meter nodes using the channel propagation equation as shown in (1). The pathloss channel coefficient is dependent on the surrounding environment. Experiments were conducted in different indoor environments to approximate the optimum value for pathloss channel coefficient:

$$
r_{i}=10^{(\mathrm{RSSI}-A) / n}
$$

where RSSI is the received signal strength indicator of power meter node $(\mathrm{dBm}), r_{i}$ range distance between $i$ th power meter node and mobile node, $n$ the pathloss coefficient of the channel, and $A$ absolute power received at a distance of $1 \mathrm{~m}$ from the transmitter $(\mathrm{dBm})$.

4.1.2. Dynamic Position Tracking Model. In order to track a person's position in real time, a fast localisation process was required. One of the drawbacks of using the trilateration RSSI-based process to localise is that the received signal strength must be averaged over a period of time. Averaged RSSI values are needed to provide suitable accuracy. However, lengthy averaging periods can lead to localisation inaccuracies. For example, a moving mobile node will distort the average received signal strength values measured by the power meter nodes.
One way to improve the accuracy of real-time tracking is to use other odometry motion information, such as a person's directional heading and speed to predict the next position of user. Similar odometry motion-based tracking models have been used for localisation tracking by Klingbeil and Wark [10] and Lau and Chung [21]. The odometry information used in [10] was estimated using motion sensors, while in [21] the speed was estimated using the previous displacement distance of the user. We implemented a similar type of DPTM used in [21], to localise a person's position by using the previous displacement and the typical human walking speed to estimate the speed of the user. The DPTM used the speed and current position to predict the person's next position. Figure 7 shows an overview of the DPTM. The DPTM can be described using the following predictive odometry motion equations concerning distance and velocity:

$$
\begin{gathered}
\hat{R}_{\mathrm{est}(i)}=\hat{R}_{\operatorname{pred}(i)}+a\left(\hat{R}_{\operatorname{prev}(i)}-\hat{R}_{\operatorname{pred}(i)}\right), \\
\hat{V}_{\mathrm{est}(i)}=\hat{V}_{\operatorname{pred}(i)}+\frac{b}{T_{S}}\left(\hat{R}_{\operatorname{prev}(i)}-\hat{R}_{\operatorname{pred}(i)}\right), \\
\hat{R}_{\operatorname{pred}(i+1)}=\hat{R}_{\mathrm{est}(i)}+\hat{V}_{\mathrm{est}(i)} \cdot T_{S}, \\
\hat{V}_{\operatorname{pred}(i)}=\hat{V}_{\mathrm{est}(i)},
\end{gathered}
$$

where $\hat{R}_{\text {est }(i)}$ is the estimated range, $\hat{R}_{\text {pred }(i)}$ predicted range, $\hat{R}_{\text {prev }(i)}$ measured range, $\hat{V}_{\text {est }(i)}$ estimated velocity, $\widehat{V}_{\text {pred }(i)}$ 
(1) Estimate current position

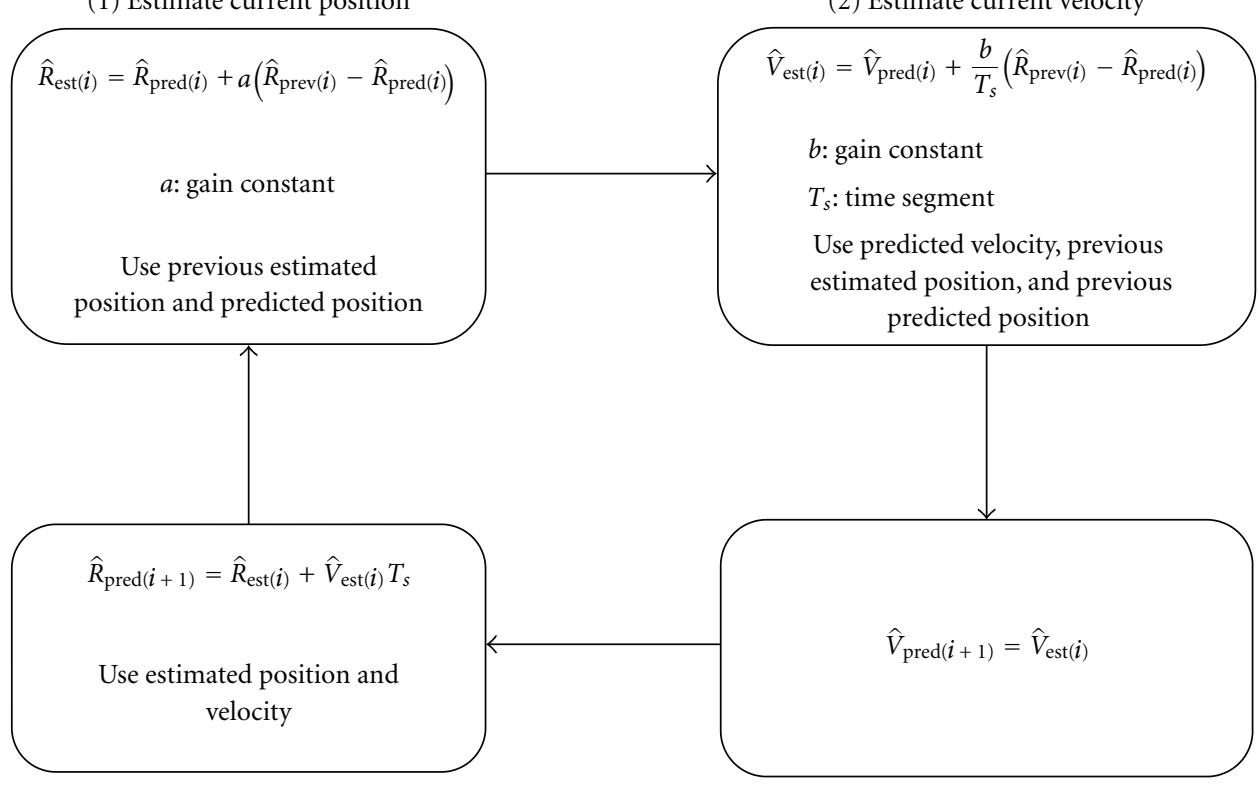

(4) Predict next position

(3) Predict next velocity

FIGURE 7: Overview of dynamic position tracking model.

predicted velocity, $a, b$ gain constants, and $T_{S}$ time update period.

The Predicted Velocity stated in (5) is an iterative process, which estimated the range using (3) to adjust the position calculated by the mobile node until the position error has been sufficiently reduced. The speed value was initially set to the average human walking speed of $1.3 \mathrm{~m} / \mathrm{s}$ (Murray et al. [22]), for the predicted range in (4). A prototype of the DPTM was implemented on the Server Computer connected to the Coordinator node.

4.1.3. Trilateration Algorithm. Once the range distances between all detected power meter nodes and the mobile node has been estimated, a trilateration algorithm is used to calculate the position of the mobile node. The trilateration algorithm used each power meter nodes coordinates and predicted range distance, calculated from the DPTM. At least 3 power meter nodes are required to be detected. If less than 3 power meter nodes are detected within the time update period, then the position and range of the last detected power meter node is used. The detection time period can vary from $10 \mathrm{~s}$ to $30 \mathrm{~s}$. The trilateration algorithm can be expressed as a typical linear system of

$$
\left[\begin{array}{c}
\left(x_{1}-x\right)^{2}+\left(y_{1}-y\right)^{2} \\
\left(x_{2}-x\right)^{2}+\left(y_{2}-y\right)^{2} \\
\vdots \\
\left(x_{n}-x\right)^{2}+\left(y_{n}-y\right)^{2}
\end{array}\right]=\left[\begin{array}{c}
r_{1}^{2} \\
r_{2}^{2} \\
\vdots \\
\vdots \\
r_{n}^{2}
\end{array}\right]
$$

$x, y$ refer to the position coordinates and $R$ is the vector of range distances between the mobile and power meter nodes. Equation (6) can be expressed as a Linear Least Squares System as seen in

$$
\left[\begin{array}{l}
x \\
y
\end{array}\right]=\left(A^{T} A\right)^{-1} A^{T} B,
$$

where $A$ and $B$ are as follows:

$$
\begin{aligned}
& A=\left[\begin{array}{cc}
\left(x_{1}-x_{n}\right) & \left(y_{1}-y_{n}\right) \\
\left(x_{2}-x_{n}\right) & \left(y_{2}-y_{n}\right) \\
\vdots & \\
\vdots & \\
\left(x_{n-1}-x_{n}\right) & \left(y_{n-1}-y_{n}\right)
\end{array}\right], \\
& B=\left[\begin{array}{c}
r_{1}^{2}-r_{n}{ }^{2}-x_{1}{ }^{2}+x_{n}{ }^{2}-y_{1}{ }^{2}+y_{n}{ }^{2} \\
r_{2}{ }^{2}-r_{n}{ }^{2}-x_{2}{ }^{2}+x_{n}{ }^{2}-y_{2}{ }^{2}+y_{n}{ }^{2} \\
\vdots \\
\vdots \\
r_{n-1}^{2}-r_{n}{ }^{2}-x_{n-1}^{2}+x_{n}{ }^{2}-y_{n-1}^{2}+y_{n}{ }^{2}
\end{array}\right]
\end{aligned}
$$

4.2. Context-Aware Tracking Algorithm. Indoor environments are characterised by unpredictable radio propagation channel parameters. These unpredictable channel parameters can cause distortion and multipath interference due to the presence of metallic structures within indoor environments. Since the trilateration algorithm uses RSSI for range 


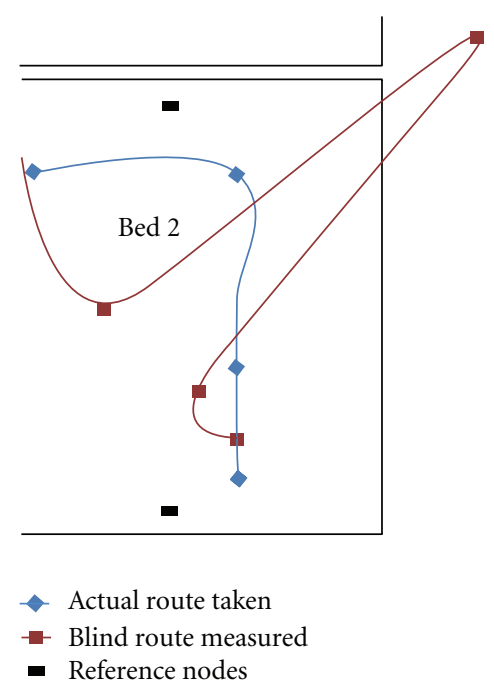

FIGURE 8: Example of an indoor navigation route predicted using RSSI-based trilateration only. Mobile node is predicted to be $0.75 \mathrm{~m}$ outside the floor plan.

estimations, wireless channel distortions will cause errors in the predicted position. An example of the influence of the RF interference can be seen in Figure 8 which compares the actual track of a mobile node with the track calculated using the trilateration position estimation. The actual path of the user was measured by walking a known surveyed path with visual observations of the current position being recorded on a regular interval by an observer. In order to overcome wireless-channel-interference-induced position errors, we developed a context-aware localisation process that used a probability density map.

The Probability Density Map (PDM) was used by the context-aware localisation process to determine the likelihood or probability of the mobile nodes estimated position. The PDM consisted of a floor plan with regions mapped with the probability of likely positions available. Position validity was approximated by detecting if the mobile node's track had to move through a wall or barrier, to its predicted position. Figure 9 shows an overview of the PDM process used to check the position of the mobile node. If the mobile node's predicted position is determined to be invalid, Least Squares approximation is used to calculate a new predicted position. The PDM process, as seen in Figure 9, is then repeated until the mobile node's position is found to be valid.

An example of the colour-coded floor plan can be seen in Figure 10. The colour red is used to highlight regions of high probability. The mobile node's position is considered to be valid if it lies in a region of high probability (red colour). The probabilities of each region are predetermined, but, in the future, this will be dynamically updated to reflect changes to the floor plan.

The wall or barrier collisions are also detected using the PDM. The barriers are considered as low probability regions (white colour) on the PDM. If the mobile node's predicted path crossed a barrier, then the DPTM would recursively reestimate the mobile node's predicted position until it becomes valid.

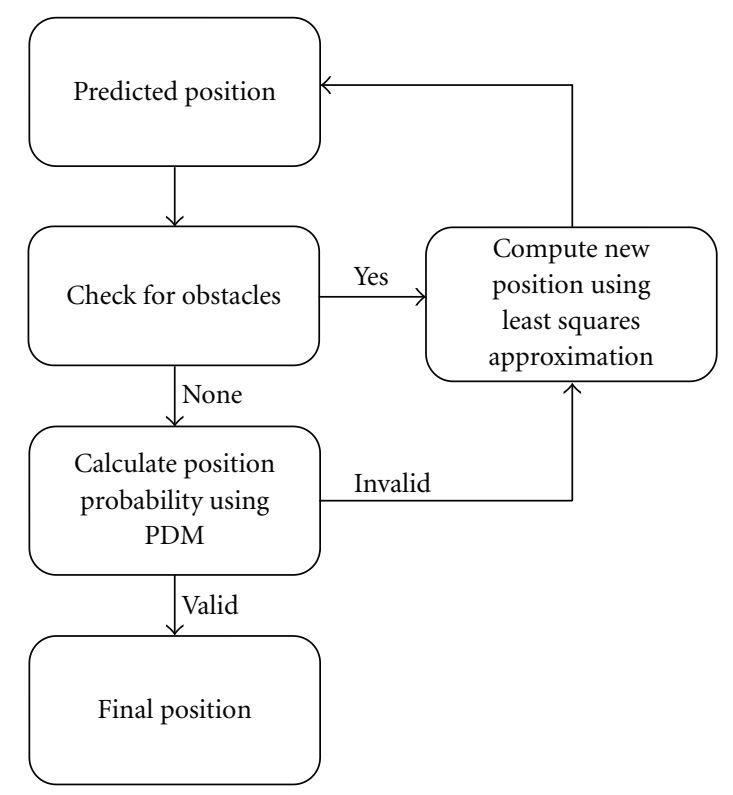

Figure 9: Overview of context-aware tracking algorithm.

\section{Evaluation}

An initial trial of the localisation network used six power meter nodes with one mobile and coordinator node. The localisation network was deployed in an indoor area of $72 \mathrm{~m}^{2}$. The aim of the trial was to evaluate the accuracy of the wireless trilateration mechanism and the context-aware tracking process. Specifically we investigated the following:

(i) the effect of wireless indoor channel propagation on position accuracy,

(ii) accuracy of the context-aware tracking model,

(iii) comparison of the context-aware tracking model accuracy with other existing wireless localisation techniques.

The localisation network of power meter nodes was deployed in a building as seen in Figure 11 on a $1 \mathrm{~m}$ spaced grid floor plan. Our tests consisted of a user walking two known paths whilst carrying a mobile node. Figure 11 shows both test paths-Route (A) and Route (B). Route (A) was $14 \mathrm{~m}$ long and was selected to have more physical obstacles such as walls, to test its effects on predicting the position of the mobile node. Route (B) was $12.5 \mathrm{~m}$ long and was selected to test how the localisation system would perform with a sparse deployment of the power meter nodes. Both routes were planned to be similar in length as to ensure that a similar distance could be walked on either routes. The ground truth or actual path of the user was measured by walking a known surveyed path with visual observations of the current position being recorded on a regular interval by an observer. We consider the test environment to be realistic for evaluating the indoor people tracker. The duration of the test path track was between 5 and 10 minutes (including time for ground truth measurements) and was repeated at least 10 times. 


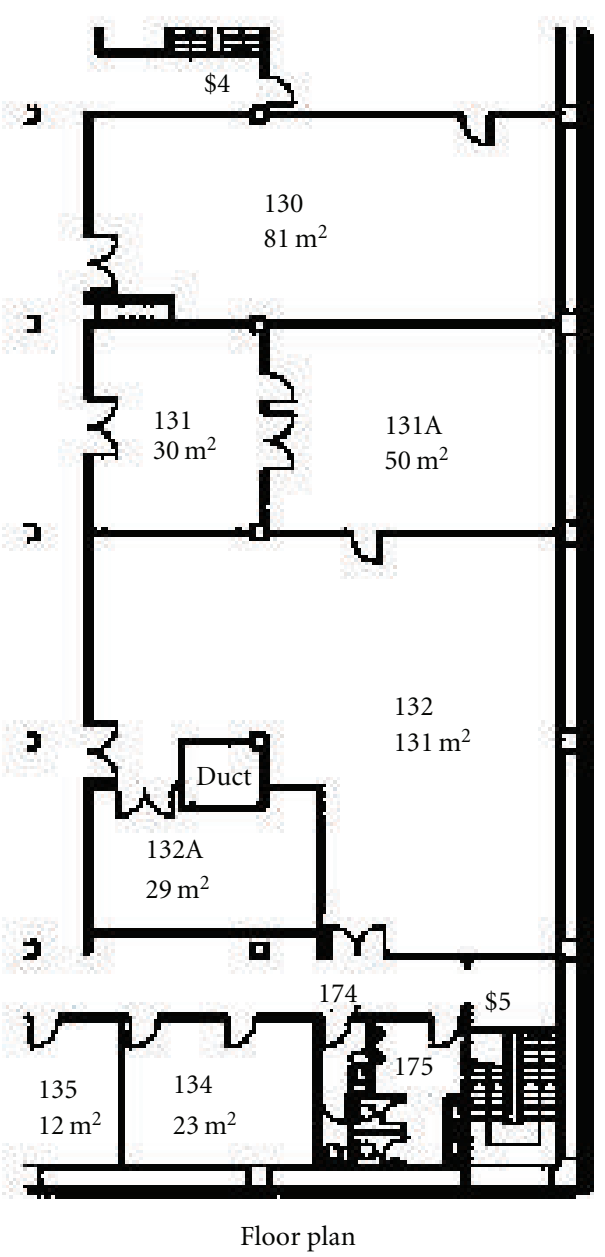

(a)

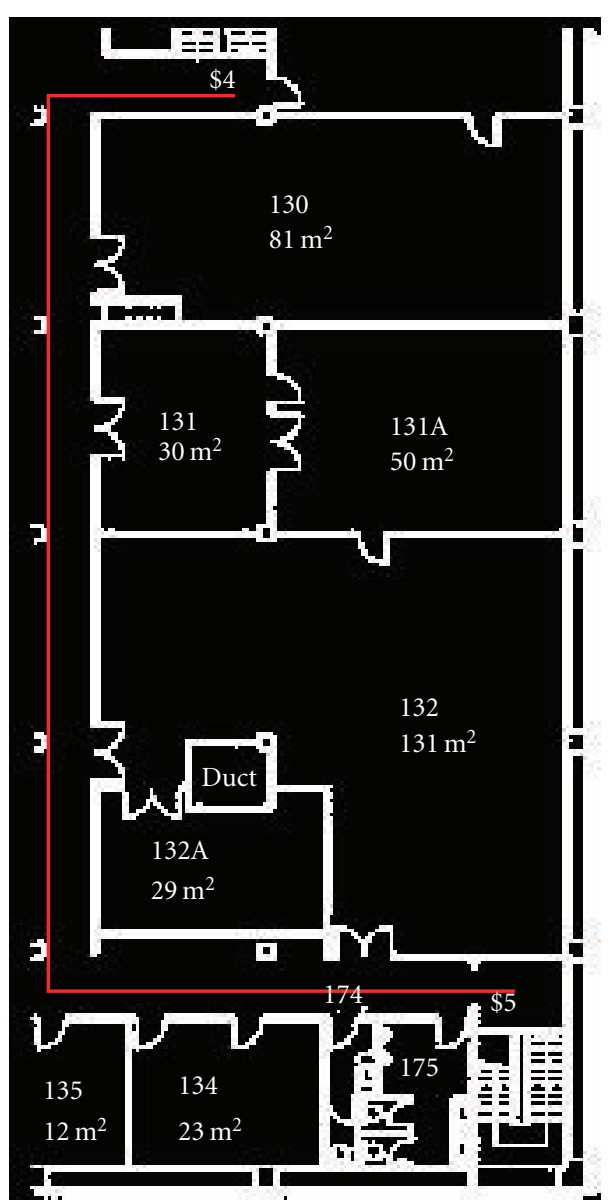

Probability density map

(b)

FIGURE 10: Example of floor plan and probability density map.

5.1. Wireless Trilateration. The wireless trilateration algorithm used the range distance estimator and dynamic position model. Figure 12 shows the predicted path tracks calculated using only wireless trilateration. Figure 13 shows the position error per distance travelled, using only the trilateration algorithm. Table 1 shows the average, minimum, and maximum position error only using the trilateration algorithm. The average position error was found to be higher for Route (B) than Route (A). This was because Route (B) had longer distances between the mobile and power meter nodes, which increased the occurrence of large range estimation errors.

5.2. Dynamic Position Tracking Model. We conducted a series of experiments to test the accuracy of the DPTM. The first experiment involved moving the mobile node in a $14 \mathrm{~m}$ straight line (not related to Route (A) or (B)) in the test deployment area at a constant walking speed. For initial testing purposes, walking in a straight line path was considered adequate to test the DPTM. The ground-truth positions of the mobile node were visually recorded by an observer. For further work, nondirect testing paths will be considered. Figure 14 shows the actual, measured, and estimated travelled distances of the mobile node over time. In Figure 14, the distance was the displacement of the mobile node from a starting point. The measure distances are calculated directly from the mobile node's coordinates (using RSSI), and the estimated distance was calculated by using the DPTM. The maximum position error was $6 \mathrm{~m}$ using the mobile nodes coordinates. Using the DPTM, the maximum position error was reduced to $3 \mathrm{~m}$.

The second experiment was similar to the first experiment except with a delay of $15 \mathrm{~s}$ introduced midway during the test. This tested how the DPTM responded to changes in movement. Figure 15 shows the actual, measured, and estimated distances with constant (continuous) velocity and changing velocity (from moving to stationary to moving again).

In order to calibrate the DPTM for good accuracy, the gain constants: $a$ and $b$, in (2) and (3) had to be optimised. Figure 15 shows the large position inaccuracy (50\%), if the gain constants $a$ and $b((2),(3))$ are not calibrated correctly. Using $a=0.06$ and $b=0.01$ was found to produce the best results. 
TABLE 1: Trilateration and context-aware tracking accuracy.

\begin{tabular}{lcccccc}
\hline & \multicolumn{3}{c}{ Route $(\mathrm{A})$} & \multicolumn{3}{c}{ Route (B) } \\
\hline Position error $(\mathrm{m})$ & Mean & Max & Min & Mean & Max & Min \\
Trilateration algorithm only & 0.91 & 2.59 & 0.22 & 2.36 & 7.49 & 0.25 \\
Context-aware tracking algorithm & 1.21 & 2.34 & 0.15 & 1.46 & 2.87 & 0.29 \\
\hline
\end{tabular}

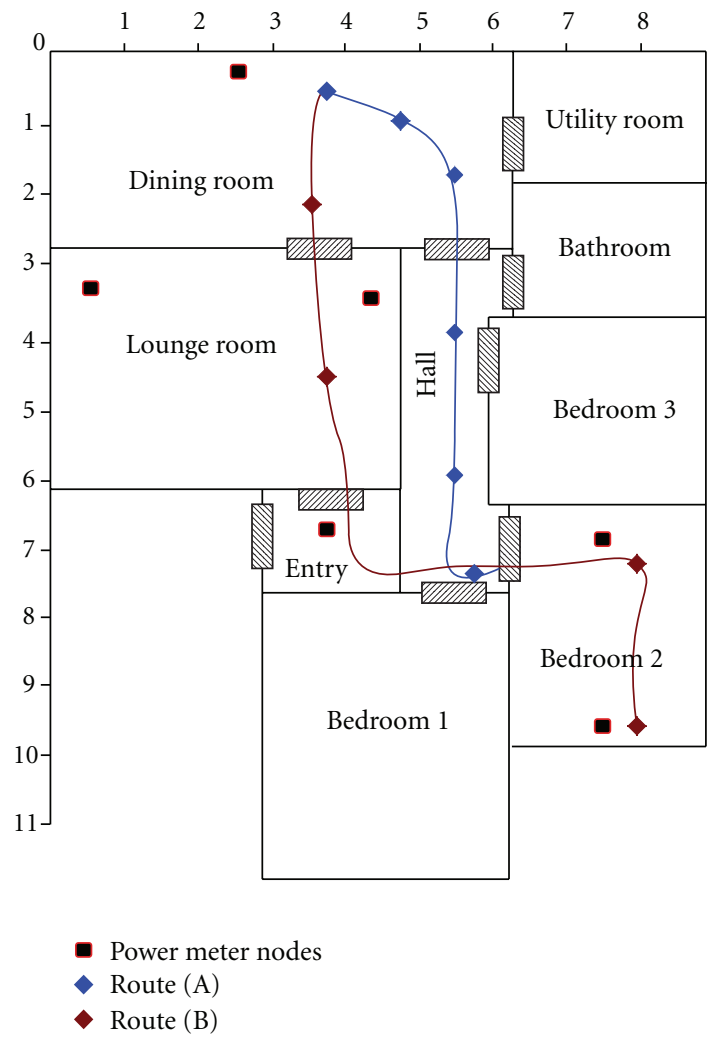

FIGURE 11: Test deployment shown on a $1 \mathrm{~m}$ spaced grid floor plan.

5.3. Context-Aware Tracking. We evaluated the contextaware tracking algorithm using the same path tracks as shown in Figure 11. Figure 12 shows the predicted path tracks calculated using the context-aware tracking algorithm. We measured the position error due to the distance travelled as seen in Figure 16. Table 1 shows the average, minimum, and maximum position error for the context-aware tracking algorithm.

As with only using the trilateration algorithm, the average position error was found to be higher for Route (B) than Route (A). Again, this was due to Route (B) having longer separation distances between the mobile and power meter nodes, which caused greater range estimation error. The context-aware algorithm compared to the trilateration estimated position had a reduced maximum position error by $9.6 \%$ for Route (A) and $67.7 \%$ for Route (B). The average position error for Route (A) was slightly higher than the trilateration estimate but was $38.56 \%$ lower for Route (B). The context-aware algorithm had a similar error for Route (A) but performed better for Route (B), when compared to the trilateration position estimates. Since the context-aware algorithm used the probability density map, it was able to compensate for range estimation errors caused by long separation distances between the mobile and power meter nodes.

5.4. Comparison with Existing Protocols. There are many wireless protocols and technologies that can be used for indoor and outdoor localisation. We compare the different RFbased protocols to the context-aware tracking system. Table 2 displays a comparison of the different RF transceiver modules used by various localisation technologies.

Although assisted GPS is known to work primarily outdoors, it is able to operate indoors. However, this requires a significant amount of additional computation. We did observe a position error of $57 \mathrm{~m}$ indoors using the SigNav subATTO TM3 assisted-GPS module [11] which can operate indoors. Wireless LAN is being used by many localisation applications, as a supplement to assisted GPS. One of the drawbacks of using Wireless LAN is the large number of hotspots required. Biswas and Veloso [23] achieved an accuracy of $0.7 \mathrm{~m}$ but required the use of Bayesian filtering which needed a significant amount of computational processing and accurate calibration. Similarly, Raghavan et al. [24] also used Bayesian filtering with Bluetooth for localisation. The drawback of Bluetooth is the long scanning time (10s-20s). Localisation using GSM was found to be $20 \mathrm{~m}$, by Otsason et al. [2]. One of the disadvantages of using GSM was its dependence on existing infrastructure.

In comparison, the context-aware tracking system consumes the least amount of power compared to the other protocols. As well, the context-aware system would require the least complex and low-powered infrastructure due to the use of wireless smart metering networks. Apart from GPS, these wireless communication protocols are designed to transfer data files rather than short messages and so require a large amount of processing power and transmit power. Hence, these protocols can be unsuitable for green pervasive computing applications.

5.5. Discussion. The context-aware tracking system was able to localise a user's position to an average error of $1.21 \mathrm{~m}$ and a maximum error of $2.34 \mathrm{~m}$ (Table 1). We have also shown that the context-aware tracking system can operate with widely and irregularly dispersed infrastructure. The advantage of the context-aware system was that it relied on wireless smart metering network infrastructure for localisation. The infrastructure for wireless smart metering is becoming more widely installed in built environments, due to the use of green energy efficiency monitoring. Compared to other systems such as Wireless LAN or GSM, using a smart 


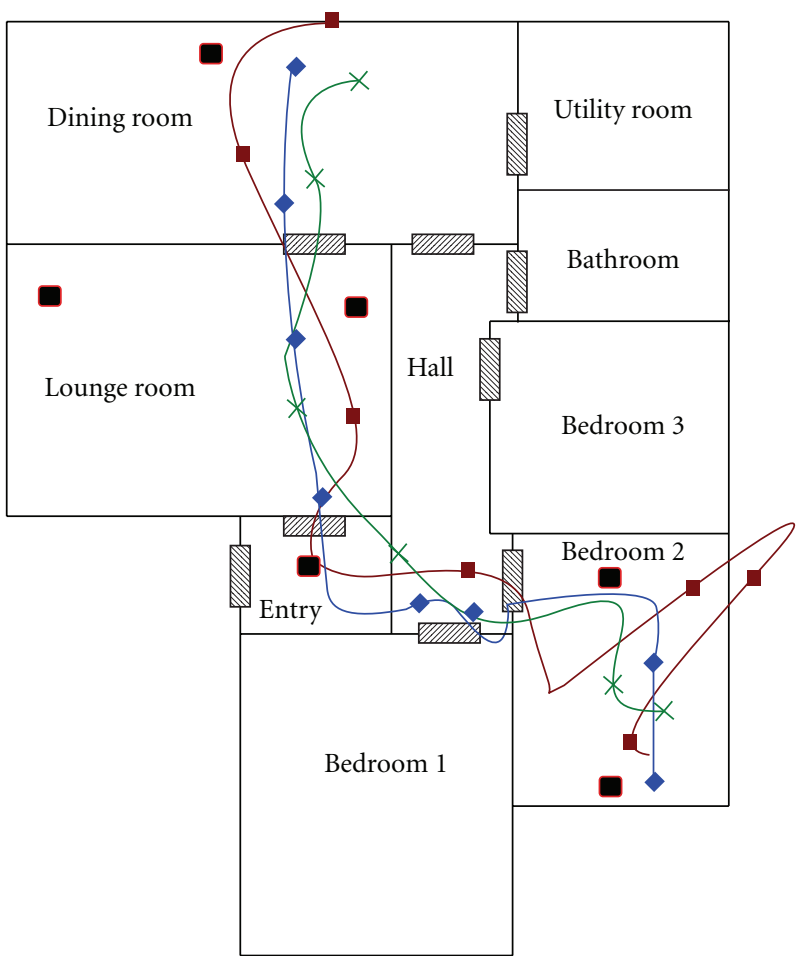

Power meter nodes Actual route taken $\times$ Context-aware algori

(a)

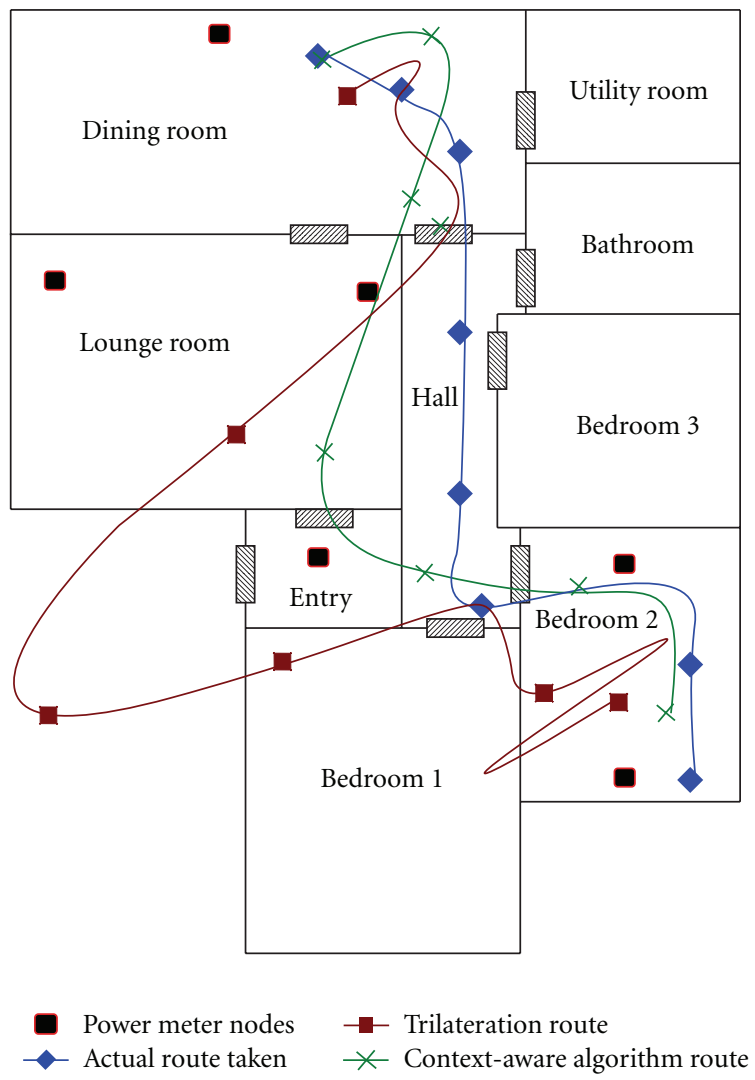

(b)

FIGURE 12: Predicted path track using the context-aware algorithm process and wireless trilateration.

TABLE 2: Comparison with assisted GPS—power and accuracy.

\begin{tabular}{|c|c|c|c|c|c|c|}
\hline Method & Accuracy-position error (m) & Latency $(s)$ & Power $(\mathrm{mW})$ & TX power $(\mathrm{mW})$ & $\begin{array}{l}\text { RX sensitivity } \\
(\mathrm{dBm})\end{array}$ & Indoor range \\
\hline Assisted GPS ${ }^{1}$ & $57 \mathrm{~m}$ & 30 & 300 & - & $-155 \mathrm{dBm}$ & Global \\
\hline $\mathrm{GSM}^{2}$ & $20 \mathrm{~m}$ & - & 1162 & 3.16 & $-14 \mathrm{dBm}$ & $200 \mathrm{~m}$ \\
\hline Wireless LAN ${ }^{3}$ & $3 \mathrm{~m}$ to $30 \mathrm{~m}$ & $11 \mathrm{~s}$ & 858 & 31.62 & $-74 \mathrm{dBm}$ & $100 \mathrm{~m}$ \\
\hline Bluetooth $^{4}$ & $3 \mathrm{~m}$ to $4 \mathrm{~m}$ & $20 s$ & 231 & 2.51 & $-88 \mathrm{dBm}$ & $30 \mathrm{~m}$ \\
\hline $\begin{array}{l}\text { Context-aware } \\
\text { tracking algorithm }\end{array}$ & $1.21 \mathrm{~m}$ (Table 1) & $2 \mathrm{~s}$ & 97.2 & $0 \mathrm{dBm}$ & $-92 \mathrm{dBm}$ & $30 \mathrm{~m}$ \\
\hline
\end{tabular}

References: ${ }^{1}[11],{ }^{2}[12],{ }^{3}[4,13],{ }^{4}[4,14]$.

metering network provides a solution that is cost effective, has low energy usage, and is easy to install.

\section{Conclusion and Further Work}

We presented a context-aware tracking system that tracked users in an indoor environment. The context-aware system used a wireless smart metering network that consisted of power meter nodes placed throughout a building. A user carried a mobile node that tracked their current position. A smartphone could be used to view the mobile nodes current position, via a cellular or wireless LAN connection.

The context-aware tracking system localised a person's position by combining wireless trilateration, a dynamic posi- tion tracking model, and a probability density map. The integral use of these three factors allowed the context-aware tracking system to achieve reasonable localisation accuracy with a sparsely and irregularly dispersed wireless network. This was advantageous compared to other RF-based localisation systems that rely on a dense wireless network to achieve good position accuracy. We found that only using wireless trilateration was not suitable for tracking users in real time due to the lengthy time required by the trilateration algorithm to overcome the effects of the wireless propagation channel. A dynamic position tracking model that estimated a position, based on a user's predicted velocity and heading, was found to improve the latency to localise a person's position. In order to further improve the position estimate, 


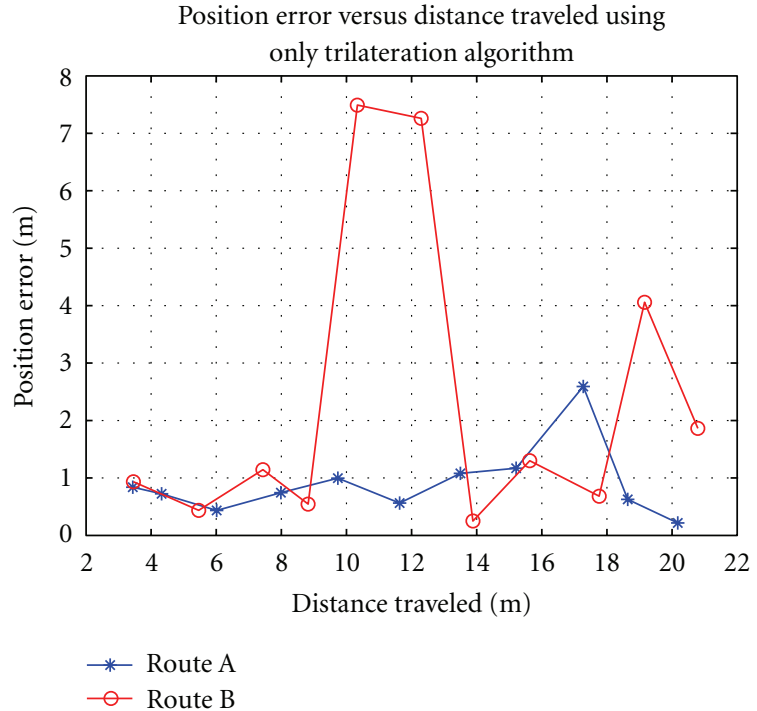

FIGURE 13: Position error versus distance traveled using only trilateration algorithm.

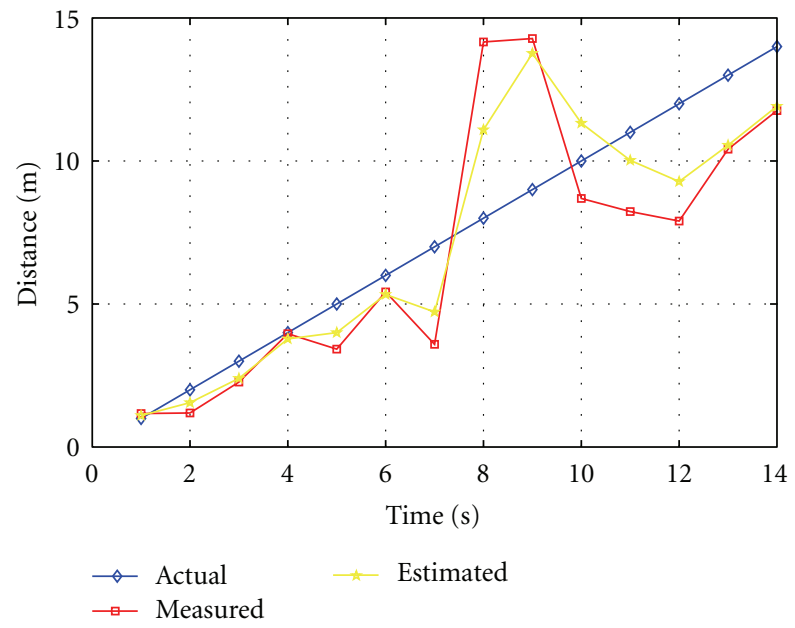

FIgURE 14: Distance versus time with a constant velocity.

a probability density map, based on the floor plan of the indoor area, was used.

An initial trial of the localisation network was conducted using six power meter nodes, a mobile and coordinator nodes. We deployed the context-aware system in an indoor space of $72 \mathrm{~m}^{2}$. We measured the channel propagation parameters and the dynamic position tracking model accuracy. We found that, by using the dynamic position tracking model, the error in position location was reduced from $6 \mathrm{~m}$ to $3 \mathrm{~m}$. Combining the use of the probability density map allowed the context-aware system to localise a person's position to an average error of $1.21 \mathrm{~m}$ and a maximum error of $2.34 \mathrm{~m}$.

The use of a wireless smart metering network for localisation is advantageous as this form of infrastructure will become more widely installed in buildings and other indoor or outdoor environments. This is due to the growing use of

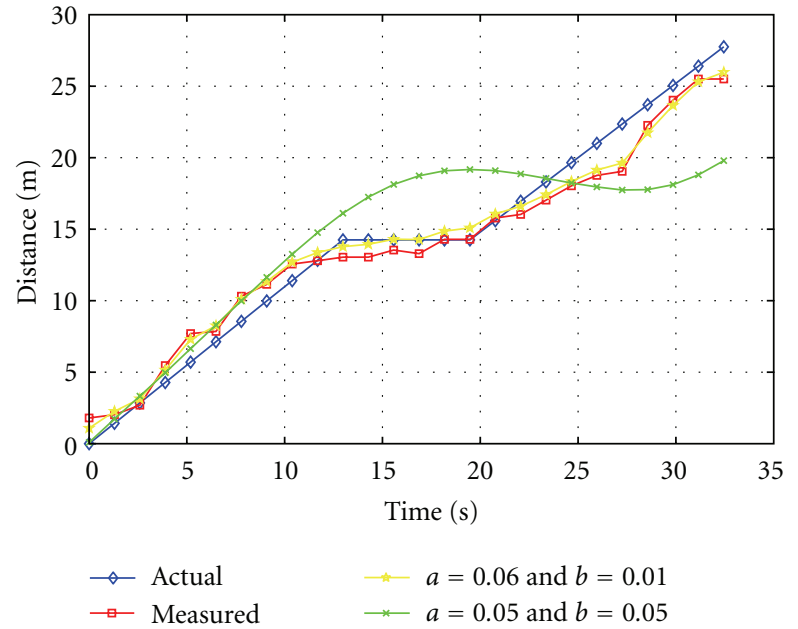

FIGURE 15: Distance versus time with a changing velocity.

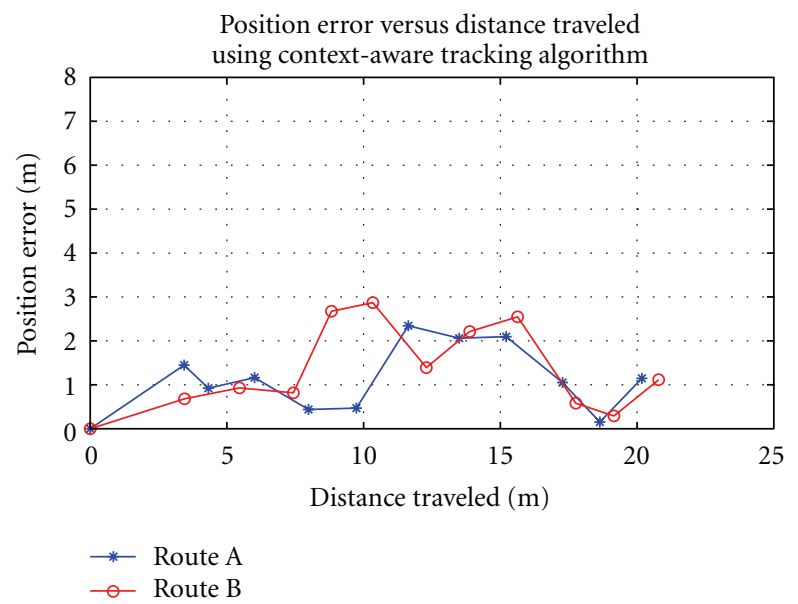

FIGURe 16: Position error versus distance traveled using contextaware tracking algorithm.

energy efficiency monitoring. Wireless smart metering infrastructure is also the least complex and low power consuming, when compared to other localisation network infrastructures. Compared to other localisation systems, the contextaware system is able to operate on little power while providing suitable accuracy for green pervasive applications.

Further work involves extensive testing with multiple operating mobile nodes and over a larger test region. Other areas of investigation involve looking at how 3-dimensional localisation can be achieved with more in-depth context awareness of the surrounding environment.

\section{References}

[1] V. Schwarz, A. Huber, and M. Tuchler, "Accuracy of a commercial UWB 3D location/tracking system and its impact on LT application scenarios," in Proceedings of the IEEE International Conference on Ultra-Wideband (ICU '05), pp. 599-603, Windisch, Switzerland, 2005. 
[2] V. Otsason, A. Varshavsky, A. LaMarca, and E. de Lara, "Accurate GSM indoor localization," in Proceedings of the International Conference on Ubiquitous Computing (UBICOMP '05), vol. 3660 of Lecture Notes in Computer Science, pp. 141-158, Tokyo, Japan, 2005.

[3] F. Seco, A. R. Jimenez, C. Prieto, J. Roa, and K. Koutsou, "A survey of mathematical methods for indoor localization," in Proceedings of the 6th IEEE International Symposium on Intelligent Signal Processing (WISP '09), pp. 9-14, Madrid, Spain, 2009.

[4] H. Liu, H. Darabi, P. Banerjee, and J. Liu, "Survey of wireless indoor positioning techniques and systems," IEEE Transactions on Systems, Man and Cybernetics Part C, vol. 37, no. 6, pp. 1067-1080, 2007.

[5] J. Hightower, A. LaMarca, and I. Smith, "Practical lessons from place lab," IEEE Pervasive Computing, vol. 5, no. 3, Article ID 1673364, pp. 32-39, 2006.

[6] R. Want, A. Hopper, V. Falcao, and J. Gibbons, "Active badge location system," ACM Transactions on Information Systems, vol. 10, no. 1, pp. 91-102, 1992.

[7] A. Ward, A. Jones, and A. Hopper, "A new location technique for the active office," IEEE Personal Communications, vol. 4, no. 5, pp. 42-47, 1997.

[8] N. B. Priyantha, A. Chakraborty, and H. Balakrishnan, "The Cricket location-support system," in Proceedings of the 6th Annual International Conference on Mobile Computing and Networking (MobiCom '00), pp. 32-43, ACM, Boston, Mass, USA, 2000.

[9] M. McCarthy, P. Duff, H. Muller, and C. Randell, "Accessible ultrasonic positioning," IEEE Pervasive Computing, vol. 5, no. 4, Article ID 1717372, pp. 86-93, 2006.

[10] L. Klingbeil and T. Wark, "A wireless sensor network for realtime indoor localisation and motion monitoring," in Proceedings of the International Conference on Information Processing in Sensor Networks (IPSN '08), pp. 39-50, St. Louis, Mo, USA, 2008.

[11] SigNav, TM3-02EH Timing Module User Guide, 2010.

[12] LinkSprite Technologies, GSM/GPRS Module User Manual, 2008.

[13] WI2WI, W2SW0001-802.11 b/g System-in-Package, 2008.

[14] Sena Technologies, OEM Bluetooth-Serial Module, ParaniESD100, 2008.

[15] S. W. Luan, J. H. Teng, S. Y. Chan, and L. C. Hwang, "Development of a smart power meter for AMI based on ZigBee communication," in Proceedings of the International Conference on Power Electronics and Drive Systems (PEDS '09), pp. 661-665, Taipei, Taiwan, 2009.

[16] Dash7 Alliance, Dash7, 2009, http://www.dash7.org/.

[17] M-Bus, "Wireless M Bus," 1999, http://www.m-bus.com/.

[18] ZigBee Alliance, ZigBee Specification, 2006, http://www.zigbee.org/.

[19] Texas Instruments, Texas instruments CC2431 zigbee development kit, 2007.

[20] Texas Instruments, CC2431 system-on-chip for 2.4 ghz zigbee/ ieee 802.15.4 with location engine, 2007.

[21] E.-E.-L. Lau and W.-Y. Chung, "Enhanced rssi-based real-time user location tracking system for indoor and outdoor environments," in Proceedings of the International Conference on Convergence Information Technology (ICCIT '07), pp. 12131218, IEEE Computer Society, 2007.

[22] M. Murray, A. Drought, and R. Kory, "Walking patterns of normal men," The Journal of Bone and Joint Surgery, vol. 46, no. 2, pp. 335-360, 1964.
[23] J. Biswas and M. Veloso, "WiFi localization and navigation for autonomous indoor mobile robots," in Proceedings of the IEEE International Conference on Robotics and Automation (ICRA '10), pp. 4379-4384, Anchorage, Alaska, USA, 2010.

[24] A. Raghavan, H. Ananthapadmanaban, M. Sivamurugan, and B. Ravindran, "Accurate mobile robot localization in indoor environments using bluetooth," in Proceedings of the IEEE International Conference on Robotics and Automation (ICRA '10), pp. 4391-4396, Anchorage, Alaska, USA, 2010. 

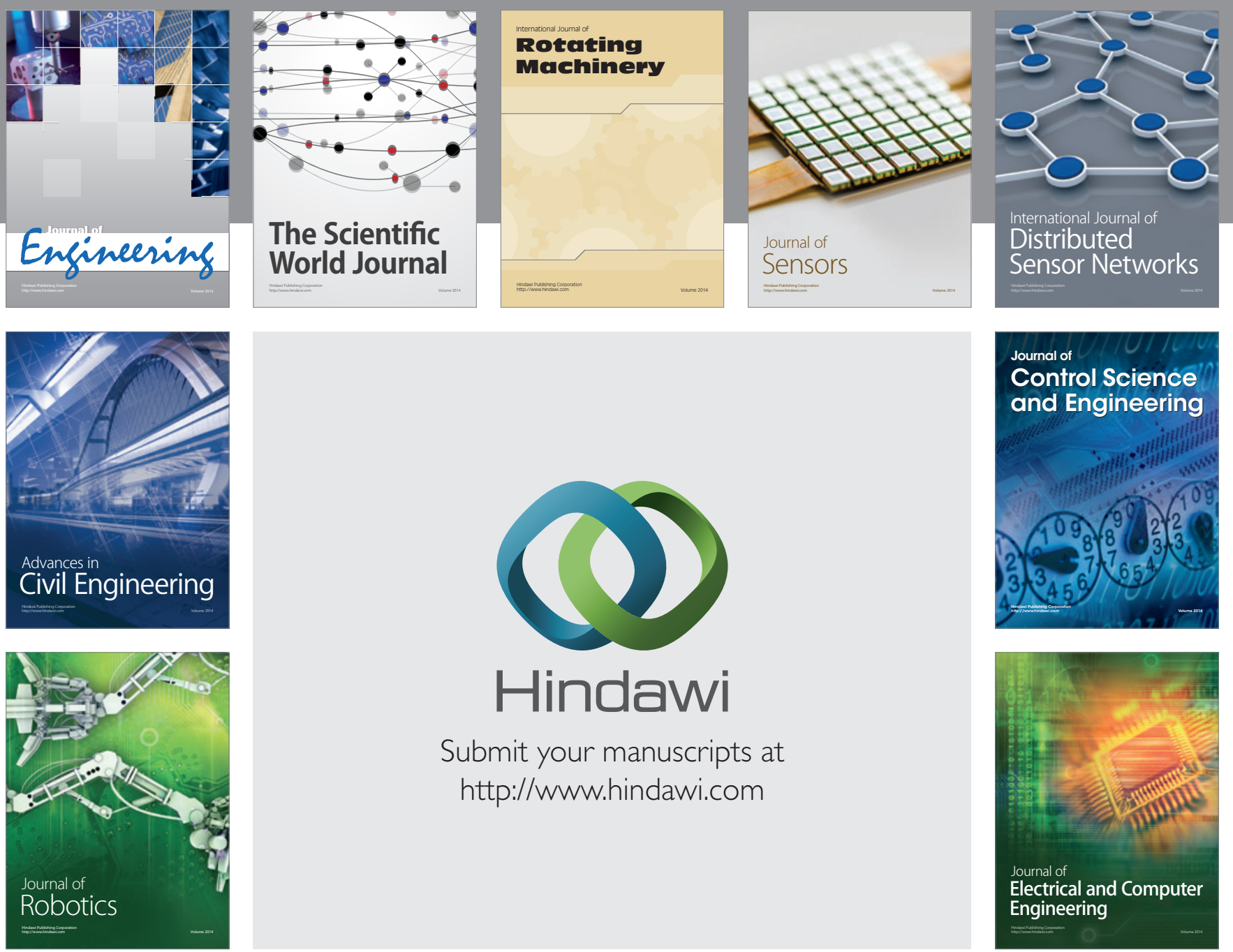

Submit your manuscripts at

http://www.hindawi.com
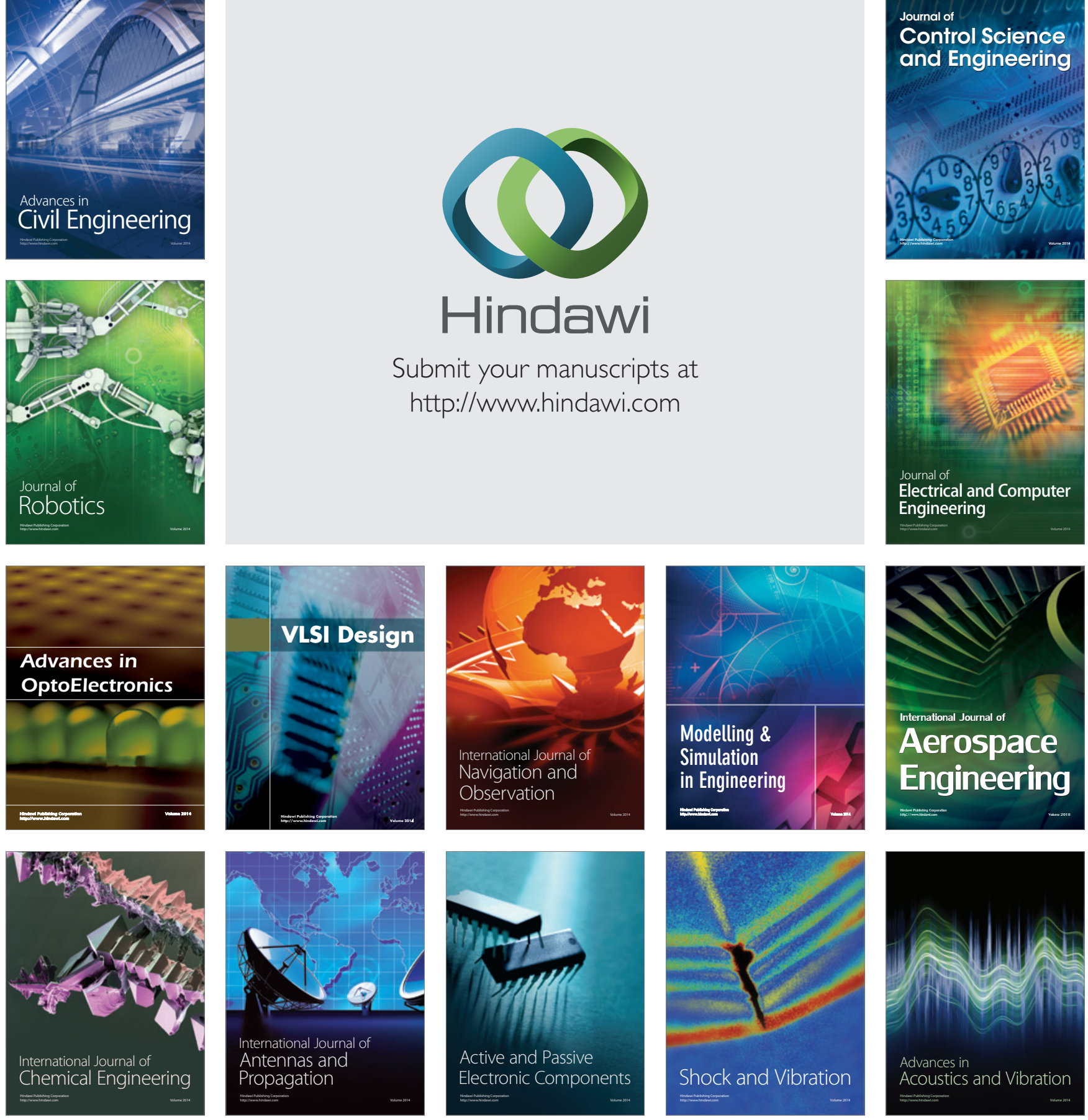